\title{
Do discrimination, residential school attendance and cultural disruption add to individual-level diabetes risk among Aboriginal people in Canada?
}

Roland F. Dyck ${ }^{1,2,3^{*}}$, Chandima Karunanayake ${ }^{1}$, Bonnie Janzen ${ }^{3}$, Josh Lawson', Vivian R. Ramsden ${ }^{4}$, Donna C. Rennie ${ }^{1}$, P. Jenny Gardipy ${ }^{5}$, Laura McCallum ${ }^{6}$, Sylvia Abonyi ${ }^{2}$, James A. Dosman ${ }^{1}$, Jo-Ann Episkenew ${ }^{7}$, Punam Pahwa ${ }^{1,2}$ and on behalf of the First Nations Lung Health Team

\begin{abstract}
Background: Aboriginal peoples in Canada (First Nations, Metis and Inuit) are experiencing an epidemic of diabetes and its complications but little is known about the influence of factors attributed to colonization. The purpose of this study was to investigate the possible role of discrimination, residential school attendance and cultural disruption on diabetes occurrence among First Nations adults.

Methods: This 2012/13 cross sectional survey was conducted in two Saskatchewan First Nations communities comprising 580 households and 1570 adults. In addition to self-reported diabetes, interviewer-administered questionnaires collected information on possible diabetes determinants including widely recognized (e.g. age, sex, lifestyle, social determinants) and colonization-related factors. Clustering effect within households was adjusted using Generalized Estimating Equations.

Results: Responses were obtained from 874 (55.7 \%) men and women aged 18 and older living in 406 (70.0 \%) households. Diabetes prevalence was $15.8 \%$ among women and $9.7 \%$ among men. In the final models, increasing age and adiposity were significant risk factors for diabetes (e.g. OR 8.72 [95 \% Cl 4.62; 16.46] for those 50+, and OR 8.97 [95 \% Cl 3.58; 22.52] for BMI 30+) as was spending most time on-reserve. Residential school attendance and cultural disruption were not predictive of diabetes at an individual level but those experiencing the most discrimination had a lower prevalence of diabetes compared to those who experienced little discrimination ( $2.4 \%$ versus $13.6 \%$; OR 0.11 [95 \% Cl 0.02; 0.50]). Those experiencing the most discrimination were significantly more likely to be married and to have higher incomes.

(Continued on next page)
\end{abstract}

\footnotetext{
* Correspondence: roland.dyck@usask.ca

${ }^{1}$ Canadian Centre for Health and Safety in Agriculture, College of Medicine, University of Saskatchewan, 104 Clinic Place, Saskatoon, Saskatchewan S7N 2Z4, Canada

${ }^{2}$ Department of Medicine, University of Saskatchewan, Saskatoon,

Saskatchewan, Canada

Full list of author information is available at the end of the article
}

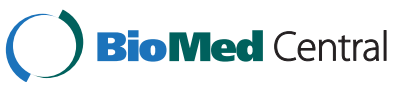

(c) 2015 Dyck et al. Open Access This article is distributed under the terms of the Creative Commons Attribution 4.0 International License (http://creativecommons.org/licenses/by/4.0/), which permits unrestricted use, distribution, and reproduction in any medium, provided you give appropriate credit to the original author(s) and the source, provide a link to the Creative Commons license, and indicate if changes were made. The Creative Commons Public Domain Dedication waiver (http://creativecommons.org/publicdomain/zero/1.0/) applies to the data made available in this article, unless otherwise stated. 
(Continued from previous page)

Conclusions: Known diabetes risk factors were important determinants of diabetes among First Nations people, but residential school attendance and cultural disruption were not predictive of diabetes on an individual level. In contrast, those experiencing the highest levels of discrimination had a low prevalence of diabetes. Although the reasons underlying this latter finding are unclear, it appears to relate to increased engagement with society off-reserve which may lead to an improvement in the social determinants of health. While this may have physical health benefits for First Nations people due to improved socio-economic status and other undefined influences, our findings suggest that this comes at a high emotional price.

Keywords: Colonization, First Nations, Residential school, Racism, Discrimination, Diabetes mellitus, Social determinants of health

\section{Background}

Aboriginal peoples in Canada (First Nations, Inuit and Metis) are experiencing an epidemic of type 2 diabetes (T2DM) [1, 2]. Compared to their nonAboriginal counterparts, First Nations people not only have higher rates of diabetes [2] but are also more likely to develop diabetes if female [2], as younger adults [2], and during childhood and adolescence [3]. Thus, while average life span is shorter among diabetic First Nations people [4] compared to non-First Nations people, the years lived with diabetes is typically longer [4]. This prolonged exposure to the metabolic consequences of diabetes contributes to a greater risk for chronic complications such as diabetic kidney disease $[5,6]$, particularly when combined with reduced access to and quality of diabetes care $[7,8]$. Understanding the mechanisms underlying these ethnicity-based differences is therefore important in developing effective primary and secondary prevention initiatives and in providing optimal management of diabetes and its complications.

While genetic factors contribute to the risk for T2DM [9], they cannot explain the rapid world-wide emergence of diabetes over the past few decades in diverse populations that include many Indigenous groups [10]. Instead, this pandemic has paralleled recent changes in environmental and possibly epigenetic factors [11] - these have been attributed to unprecedented disruptions in traditional lifestyles that have occurred in virtually all human populations, particularly since the middle of the past century [12]. Most attention has focused on the role of changing diets (e.g. higher consumption of simple carbohydrates and calorie dense foods) and reduced physical activity that have led to a parallel pandemic of overweight/obesity [13]. Indeed, overweight/obesity is an important diabetes risk factor among First Nations people which is compounded by a higher proportion with elevated body mass index (BMI) [14]. There is also mounting evidence that increasing rates of childhood obesity and T2DM are partly driven by an increased incidence of diabetic pregnancies among First Nations women not only women with gestational diabetes [15] but also their children [16] have a higher risk for T2DM.

In addition to changes in lifestyle, it has become apparent that inequities in the social determinants of health (e.g. poverty, sub-standard housing, low educational attainment, poor food availability, unemployment) [17] are also important predictors of chronic diseases including diabetes [18], and are widespread in Aboriginal communities [19]. These factors are increasingly attributed to the impact of colonization that has severely undermined Indigenous culture and access to resources necessary to improve socio-economic status [20, 21]. In particular, discrimination has been identified as a potent social stressor that may increase vulnerability to physical illness through physiological, psychological and behavioral pathways [22]. The main purpose of this study was to determine if residential school attendance, indicators of cultural disruption and perceptions of discrimination were individual-level predictors of diabetes among adults in two First Nations communities in Canada after adjusting for other recognized diabetes risk factors.

\section{Methods}

This was a cross-sectional survey that examined the prevalence, co-morbidities and both widely recognized and unique predictors of diabetes mellitus among First Nations adults living in two Cree First Nations communities in central Saskatchewan in 2012 and 2013. The study was conducted as part of the First Nations Lung Health Project (FNLHP) that used a community-based participatory research approach. The methodology for the overall study has been described in detail elsewhere [23], but its main features and the specific elements of this research are also summarized here.

The overall project was initially developed through two years of dialogue and ten consultation sessions with community leaders, health workers and community members. Thereafter, a Decision Makers Council consisting of band councilors, elders and youth was formed 
to oversee the FNLHP. We received letters of agreement from the two communities that included a commitment to follow the Canadian Institutes for Health Research (CIHR) guidelines for conducting research in Aboriginal communities. Regular updates with community representatives are ongoing and all findings are shared with community leaders with agreement to go forward. We have conducted 8 focus groups with communities to share findings and have had 2 national meetings on housing and health led by community leaders. Before conducting the survey, the Biomedical Research Ethics Board of the University of Saskatchewan approved the study (Certificate No. Bio \#12-89) and written consent was obtained from all participants.

In developing the baseline questionnaires, feedback was first obtained from community advisors (Elders and health services directors/staff) of both participating communities. Advisors also provided input about the best approaches for contacting participants and for collecting questionnaire and clinical assessment data. A pilot study was conducted to optimize the content and administration of the baseline questionnaires. Based on the pilot project responses, several questions were modified in the final survey. In both First Nations communities, the baseline assessment consisted of two stages carried out by trained community research assistants. The first stage involved door-to-door canvassing to advertise the survey by distributing brochures and explaining the need and purpose of the study. For the second stage, every adult (18 years and older) was invited to visit the community health care center to complete intervieweradministered questionnaires and to participate in clinical assessments [23] (height, weight, waist circumference, blood pressure, pulmonary function and allergy skin prick tests). Finally, an identification number was assigned to each household using the community map, and a key informant from each household was asked to provide household level information. The sampling frame and sample size calculations can be found in our Methods paper [23].

\section{Primary health outcome}

The questionnaires were primarily designed to obtain information on the individual and contextual determinants of respiratory health, but also included questions pertaining to general health and other health conditions. Accordingly, the outcome of interest for this study was self-reported physician-diagnosed diabetes mellitus. We did not attempt to distinguish between type 1 and type 2 diabetes.

\section{Contextual factors}

The contextual factors of interest in this sub-study study were: housing environment, socioeconomic status, household income, and factors that can be attributed to colonization [20, 21]. This was approached by asking questions about knowledge of Indigenous language (Cree), participation in traditional cultural activities, scoring of community strengths (scale 1-12), residential school experience [24] (either personally or by family members), and perceptions of discrimination. The latter variable was created by summing the affirmative responses to 11 situations modified from the 9 item scale published by Krieger [25-27] (Have you ever experienced discrimination or racism, been prevented from doing something, or been hassled or made to feel inferior in any of the following situations because of your race, cultural group or color: at school; getting hired or getting a job; at work; getting housing; getting medical care; getting medicine at pharmacies; getting service in a store or restaurant; getting credit, bank loans or mortgage; on the street or public setting; from the police or the courts; getting a check cashed). Affirmative responses were divided into three categories of perceived discrimination: low (0-3), moderate (4-7), high (8-11). These differed from Krieger's cut-offs $(0,1$ or 2, 3-9 respectively) because of the additional 2 items (getting medicine at pharmacies; getting a check cashed) and to more clearly identify those experiencing the most discrimination.

Following an extensive review of existing measures of discrimination, we chose the Krieger scale because of its established psychometric properties and its brevity compared to similar scales. Although extensive validation procedures were not conducted (as we did not have the data to do so), pilot testing of the instrument indicated that the scale items resonated with community members. Furthermore, to further enhance the content validity of the scale, two items were added based on community feedback (see above). We were also able to determine that the scale had high internal consistency in our sample (Cronbach alpha $=.87$ ), an important component when assessing the validity of a scale. Nonetheless, additional research is needed to further examine its' validity and reliability in relation to the experiences of Indigenous people in a Canadian context.

\section{Individual factors}

The individual factors considered were: lifestyle or behavior-related factors (smoking, alcohol use, body mass index (BMI); waist circumference, and physical activity); and individual educational attainment.

\section{Covariates}

Information was obtained on important covariates such as age, sex and marital status. There were also questions pertaining to perceptions of general health as well as selected co-morbid conditions including depression, 
hypertension, macro-vascular disease (heart disease, stroke, leg ulcers, amputation), micro-vascular disease (eye sight problems and kidney disease), tuberculosis, cancer and several indicators of chronic lung disease.

\section{Statistical analysis}

Statistical analyses were conducted using SPSS version 22 (SPSS Inc. Armonk, NY: IBM Corp.). For baseline survey data, frequencies were computed for all variables. Both crude and age specific diabetes prevalence were presented as observed/total and percentage. Chi-square tests were used to determine the univariate association of diabetes prevalence with the independent variables of interest. Logistic regression models were used to predict the relationship between a binary diagnosis of diabetes (yes or no) and a set of explanatory variables. A multilevel logistic regression modeling approach based on a generalized estimating equations, with individuals (1st level) nested within households (2nd level), was utilized to evaluate the effects of both contextual and individual factors after adjustment for covariates of interest. This accounts for the within subject dependencies that occur in the analysis due to multiple people from the same household. A series of multi-level models were fitted to determine whether potential risk factors, confounders, and interactive effects (e.g. individual and contextual risk factors) contributed significantly to the prevalence of diabetes. Based on bi-variable analysis, variables with $p$ $<0.20$ were candidates for the multivariate model. All variables that were statistically significant $(p<0.05)$ as well as important contextual factors (discrimination), were retained in the final multivariable model. Interactions between potential effect modifiers were examined and were retained in the final model if the $\mathrm{p}$-value was $<0.05$. The predicted probability graphs were prepared for significant interactions. A parsimonious model was selected based on QIC (Quasi likelihood under the Independence model Criteria) goodness-of-fit statistic $[28,29]$. The strengths of associations were presented by odds ratios (OR) and their $95 \%$ confidence intervals $(\mathrm{CI})$.

\section{Results}

Of the 1570 eligible adults (18 years of age and older) and 580 eligible households in the two First Nations communities, 874 $(55.7 \%)$ individuals living in 406 (70 \%) households participated in the survey (Table 1). Of those, 443 (51\%) were female and 431 (49\%) were male; their mean age was 35 years (range 18-85 plus 1 person aged 17). There were 112 people with diabetes (42 males and 70 females). The overall crude diabetes prevalence was $12.8 \%$ with a higher proportion of affected females than males (15.8 \% versus $9.7 \%)$. The
Table 1 Study population characteristics - adults 18 and older ${ }^{a}$

\begin{tabular}{lll}
\hline Parameters & Number & Percent \\
\hline Eligible Households & 580 & - \\
Participating Households & 406 & 70 \\
Eligible Adults & 1570 & - \\
Participating Adults (total) & 874 & 55.7 \\
Men & 431 & 49.3 \\
Women & 443 & 50.7 \\
Total self-reported diabetes & 112 & 12.8 \\
Men & 42 & 9.7 \\
Women & 70 & 15.8 \\
Diabetes by Age Group (Total) & & \\
50 and older & $56(172)$ & 32.6 \\
$40-49$ & $23(135)$ & 17.0 \\
$30-39$ & $19(175)$ & 10.9 \\
Under 30 & $14(392)$ & 3.6 \\
\hline
\end{tabular}

The age/sex distribution of the study population closely approximated the 2011 Canadian census for these two communities

age-specific prevalence ranged from $3.6 \%$ in those under 30 years of age to $32.6 \%$ among those 50 and older.

Table 2 shows the univariate relationships between key variables and diabetes. Those with diabetes were more likely to be female, older, divorced/separated/widowed, and to be retired/homemaker/disabled. Single people and those who were either employed or students were less likely to have diabetes. In this study, lower educational attainment and reduced household income were not predictors of diabetes although the prevalence was significantly higher among people living in houses requiring major repairs. First Nations people who were overweight, obese or had an increased abdominal girth were respectively 5.9, 14.5 and 8.5 times more likely to have diabetes than those with normal adiposity. Regular alcohol intake and being a current smoker both significantly reduced diabetes risk in univariate analysis but not after adjusting for other independent variables. There was a trend for exercise to reduce diabetes risk in a dose-dependent manner but this relationship was not statistically significant.

Table 3 shows the relationship between diabetes and factors that can be attributed to colonization. Diabetes prevalence was slightly higher among participants who had attended residential schools than those who had not (13.5\% versus $12.4 \%$ ) but the difference was not statistically significant (OR 1.10; 95 \% CI 0.74, 1.66). Similarly, having a parent or grandparent who had attended residential school did not significantly predict diabetes among study participants, although the overall number of community members who did not have an intergenerational family exposure to residential schools was $<10 \%$. Additional analyses (not shown) that examined 
Table 2 Univariate associations between diabetes and individual characteristics

\begin{tabular}{|c|c|c|c|c|c|}
\hline Parameter & Diabetes Prevalence (\%) & Odds Ratio (95 \% Cl) & Parameter & Diabetes Prevalence (\%) & Odds Ratio (95 \% Cl) \\
\hline Sex & & & Life Style & & \\
\hline Female & 15.8 & $1.73(1.16,2.58)$ & Body Mass Index & & \\
\hline \multirow[t]{2}{*}{ Male } & 9.7 & 1.00 (ref) & Obese & 23.8 & $14.5(6.09,34.61)$ \\
\hline & & & Overweight & 11.2 & $5.86(2.40,14.35)$ \\
\hline Age & & & Normal & 2.1 & 1.00 (ref) \\
\hline $50+$ years & 32.6 & $13.5(7.27,24.98)$ & & & \\
\hline 40-49 years & 17.0 & $5.63(2.79,11.35)$ & Abdominal Girth ${ }^{a}$ & & \\
\hline 30-39 years & 10.9 & $3.39(1.69,6.78)$ & High risk & 19.2 & $8.50(4.06,17.79)$ \\
\hline \multirow[t]{2}{*}{$17-29$ years } & 3.6 & 1.00 (ref) & Not available & 16.1 & $5.73(2.35,13.97)$ \\
\hline & & & Low risk & 2.7 & 1.00 (ref) \\
\hline \multicolumn{6}{|l|}{ Marital Status } \\
\hline Single & 8.4 & $0.47(0.31,0.74)$ & Smoking Status & & \\
\hline Divorced/separated/widowed & 29.8 & $2.19(1.21,3.94)$ & Current smoker & 10.9 & $0.53(0.30,0.95)$ \\
\hline \multirow[t]{2}{*}{ Married/common law } & 16.3 & 1.00 (ref) & Ex-smoker & 20.2 & $1.10(0.56,2.15)$ \\
\hline & & & Never Smoker & 18.6 & 1.00 (ref) \\
\hline \multicolumn{6}{|l|}{ Education Attained } \\
\hline Less than high school & 12.8 & $0.99(0.66,1.49)$ & Alcohol Intake & & \\
\hline \multirow[t]{2}{*}{ High school or higher } & 12.9 & 1.00 (ref) & Regular & 8.8 & $0.58(0.35,0.98)$ \\
\hline & & & Never or not regular & 14.2 & 1.00 (ref) \\
\hline \multicolumn{6}{|l|}{ Household income } \\
\hline$\$ 0-\$ 9,999$ & 12.2 & $0.92(0.55,1.56)$ & Exercise & & \\
\hline$\$ 10,000-\$ 19,999$ & 12.6 & $0.96(0.49,1.85)$ & Yes & 11.9 & $0.78(0.51,1.19)$ \\
\hline Refused/Not stated & 13.2 & $1.01(0.60,1.69)$ & No & 14.9 & 1.00 (ref) \\
\hline \multirow[t]{2}{*}{$\$ 20,000+$} & 13.0 & 1.00 (ref) & & & \\
\hline & & & Duration of Exercise & & \\
\hline Employment Status & & & $0-<15$ minutes & 15.1 & $1.16(0.66,2.05)$ \\
\hline Employed (full/part/student) & 9.6 & 1.00 (ref) & 15-30 minutes & 10.8 & $0.79(0.42,1.50)$ \\
\hline Retired/homemaker/disabled & 20.2 & $2.38(1.41,4.04)$ & $31-60$ minutes & 9.8 & $0.71(0.36,1.40)$ \\
\hline Unemployed & 11.6 & $1.23(0.74,2.04)$ & $>60$ minutes & 13.3 & 1.00 (ref) \\
\hline House Environment & & & Sleep Hours & & \\
\hline Crowding & & & More than 7 hours & 11.4 & $0.77(0.43,1.40)$ \\
\hline$<1$ person/bedroom & 19.5 & $1.87(1.04,3.36)$ & $6-7$ hours & 14.0 & $0.99(0.56,1.76)$ \\
\hline 1 person/bedroom & 15.5 & $1.41(0.85,2.34)$ & 5 hours or less & 14.3 & 1.00 (ref) \\
\hline$>1$ person/bedroom & 11.5 & 1.00 (ref) & & & \\
\hline \multicolumn{6}{|l|}{ House needs repair } \\
\hline Yes, major repairs & 15.7 & $1.66(1.00,2.74)$ & & & \\
\hline Yes, minor repairs & 12.5 & $1.28(0.72,2.25)$ & & & \\
\hline No, only regular maintenance & 10.4 & 1.00 (ref) & & & \\
\hline
\end{tabular}

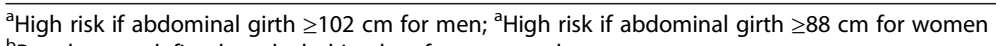

${ }^{\mathrm{b}}$ Regular was defined as alcohol intake of once a week or more

the relationship between diabetes and residential school attendance by sex, age group, micro/macro-vascular complications and community, also revealed no statistically significant relationships. The degree to which people participated in cultural events or perceived community strength were also neither predictive nor protective in their relationship with diabetes. In contrast, those who spoke Cree or lived exclusively in their home communities were significantly more likely to have diabetes than those who spent some time off-reserve. 
Table 3 Univariate associations between diabetes and factors related to colonization

\begin{tabular}{|c|c|c|c|c|c|}
\hline Parameter & Diabetes Prevalence (\%) & Odds Ratio (95 \% Cl) & Parameter & Diabetes Prevalence (\%) & Odds Ratio (95 \% Cl) \\
\hline \multicolumn{6}{|l|}{$\begin{array}{l}\text { Residential School } \\
\text { Attendance }\end{array}$} \\
\hline Study participant? & & & $\begin{array}{l}\text { Take part in cultural } \\
\text { events? }\end{array}$ & & \\
\hline Yes & 13.5 & $1.10(0.74,1.66)$ & Always/almost always & 12.3 & $1.08(0.45,2.60)$ \\
\hline \multirow[t]{2}{*}{ No } & 12.4 & 1.00 (ref) & Sometimes & 14.3 & $1.29(0.60,2.78)$ \\
\hline & & & Rarely & 9.8 & $0.84(0.34,2.12)$ \\
\hline Parents/grandparents? & & & Never & 11.4 & 1.00 (ref) \\
\hline Yes & 12.2 & $0.65(0.36,1.19)$ & & & \\
\hline Do not know & 13.3 & $0.72(0.28,1.85)$ & Community Strength $^{b}$ & & \\
\hline \multirow[t]{2}{*}{ No } & 17.4 & 1.00 (ref) & Low & 14.2 & $1.28(0.85,1.95)$ \\
\hline & & & High & 11.4 & 1.00 (ref) \\
\hline \multicolumn{6}{|c|}{ Experience discrimination $^{a}$} \\
\hline High (8-11) & 2.4 & $0.15(0.03,0.65)$ & $\begin{array}{l}\text { Past Year Spent on } \\
\text { Reserve }\end{array}$ & & \\
\hline Moderate (4-7) & 15.1 & $1.15(0.70,1.87)$ & All 12 months & 15.5 & $2.46(1.48,4.08)$ \\
\hline Little (0-3) & 13.6 & 1.00 (ref) & Less than 12 months & 7.0 & 1.00 (ref) \\
\hline \multicolumn{6}{|l|}{ Language } \\
\hline Can speak Cree & 21.2 & $3.65(2.37,5.64)$ & & & \\
\hline Cannot speak Cree & 6.9 & 1.00 (ref) & & & \\
\hline
\end{tabular}

${ }^{a}$ This variable was created by summing up the affirmative responses to 11 situations (see methods)

${ }^{b}$ This variable was created by summing up the affirmative responses to 12 strengths in the community. If the study participant perceived less than or equal to 4 community strengths it was coded as low, and if greater than 4 community strengths it was coded as high

However, ability to speak Cree was not a significant predictor of diabetes after adjusting for other independent variables.

Table 3 also shows the association between perceptions of discrimination and diabetes prevalence. Those experiencing the highest level of discrimination had the lowest prevalence of diabetes when compared to those experiencing little discrimination (2.7\% versus $13.6 \%$; OR 0.15 ; $95 \%$ CI 0.03, 0.55). Those exposed to a moderate level of discrimination had a slightly higher prevalence of diabetes than the reference group but the difference was not statistically significant.

The univariate associations between diabetes and several important co-morbidities are shown in Table 4. Overall, people with diabetes were 3.6 times more likely to perceive their general health as fair and over 4 times as likely to perceive it as poor compared to those without diabetes. These perceptions paralleled the presence of micro-vascular and macro-vascular disease (ORs 3.63 and 4.25 respectively). People with diabetes were also more likely to report a significant history of cancer, tuberculosis and asthma. Although chronic respiratory diseases and obstructive sleep apnea were more prevalent in people with diabetes, the findings did not achieve statistical significance. Nonetheless, symptoms such as shortness of breath, snoring and abnormal sleepiness were significantly associated with diabetes, as were several tests indicating poorer lung function. Finally, there was a trend for people with diabetes to have higher blood pressures, mental health problems and depression but once again the findings were not statistically significant.

Tables 5 show results from two models of diabetes risk based on multivariate logistic regression analysis. The first was carried out with and without BMI included in the model; and the second with and without abdominal girth. In both models the results were similar. Increasing age and increasing adiposity (either BMI or high risk abdominal girth) were highly predictive of diabetes when measures of adiposity were included in the model, while female sex was not. In contrast, when BMI and abdominal girth were removed from the model, women were over $50 \%$ more likely to have diabetes than men (OR 1.54; 95 \% CI 1.00, 2.38).

As in univariate analysis (above), place of habitation continued to be a significant predictor of diabetes in 3 of the 4 multivariate models. Thus, those living on-reserve for all 12 months of the year were more likely to have diabetes than those who spent at least part of the time off-reserve. Finally, those experiencing the highest levels 
Table 4 Univariate associations between diabetes and co-morbidities

\begin{tabular}{|c|c|c|c|c|c|}
\hline Parameter & Diabetes Prevalence (\%) & Odds Ratio (95 \% Cl) & Parameter & Diabetes Prevalence (\%) & Odds Ratio $(95 \% \mathrm{Cl})$ \\
\hline Shortness of Breath & & & Tuberculosis & & \\
\hline Yes & 16.7 & $2.17(1.40,3.36)$ & Yes & 22.0 & $2.08(1.07,4.03)$ \\
\hline No & 8.5 & 1.00 (ref) & No & 12.0 & 1.00 (ref) \\
\hline Asthma & & & Sleep Apnea & & \\
\hline Yes & 19.5 & $1.87(1.17,2.98)$ & Yes & 19.4 & $1.73(0.89,3.36)$ \\
\hline No & 11.4 & 1.00 (ref) & No & 12.2 & 1.00 (ref) \\
\hline General Physical Health & & & $\begin{array}{l}\text { Chronic Respiratory } \\
\text { Diseases }{ }^{c}\end{array}$ & & \\
\hline Poor & 22.7 & $4.11(1.19,14.26)$ & Yes & 21.2 & $1.95(0.96,3.99)$ \\
\hline Fair & 20.5 & $3.61(1.28,10.20)$ & No & 12.1 & 1.00 (ref) \\
\hline Good & 11.3 & $1.78(0.61,5.17)$ & & & \\
\hline Very good & 7.3 & $1.10(0.34,3.54)$ & Snore & & \\
\hline \multirow[t]{2}{*}{ Excellent } & 6.7 & 1.00 (ref) & Yes & 15.2 & $1.64(1.07,2.53)$ \\
\hline & & & Do not know & 12.3 & $1.28(0.71,2.31)$ \\
\hline Mental Health & & & No & 9.9 & 1.00 (ref) \\
\hline Fair to poor & 18.5 & $1.68(0.98,2.89)$ & & & \\
\hline \multirow[t]{2}{*}{ Excellent to good } & 11.9 & 1.00 (ref) & Snore Volume & & \\
\hline & & & Loud to very loud & 21.0 & $2.43(1.41,4.19)$ \\
\hline Depression & & & Softer than talking & 10.6 & $1.08(0.59,1.98)$ \\
\hline Yes & 16.8 & $1.54(0.95,2.50)$ & Do not know & 12.9 & $1.35(0.81,2.26)$ \\
\hline No & 11.5 & 1.00 (ref) & Never & 9.9 & 1.00 (ref) \\
\hline Macro Vascular Complications ${ }^{a}$ & & & $\begin{array}{l}\text { Epworth Sleepiness } \\
\text { Scale }\end{array}$ & & \\
\hline Yes & 32.1 & $4.25(2.67,6.77)$ & Abnormal & 17.6 & $1.73(0.99,3.03)$ \\
\hline No & 10.1 & 1.00 (ref) & Normal & 11.0 & 1.00 (ref) \\
\hline Micro Vascular Complications ${ }^{\text {b }}$ & & & $\begin{array}{l}\text { Systolic Blood } \\
\text { Pressure }\end{array}$ & & \\
\hline Yes & 29.0 & $3.63(2.33,5.66)$ & High & 15.4 & $1.44(0.93,2.22)$ \\
\hline \multirow[t]{2}{*}{ No } & 10.1 & 1.00 (ref) & Not Available & 12.7 & $1.16(0.59,2.29)$ \\
\hline & & & Low & 11.3 & 1.00 (ref) \\
\hline \multicolumn{6}{|l|}{ Cancer } \\
\hline Yes & 31.0 & $3.32(1.49,7.40)$ & & & \\
\hline No & 11.9 & 1.00 (ref) & & & \\
\hline Lung function measurements & Mean \pm SD & Odds Ratio (95 \% Cl) & & & \\
\hline \multicolumn{6}{|l|}{ FVC } \\
\hline Diabetes-Yes & $3.8 \pm 1.0$ & $0.45(0.34,0.60)$ & & & \\
\hline Diabetes-No & $4.6 \pm 1.1$ & 1.00 (ref) & & & \\
\hline \multicolumn{6}{|l|}{$\mathrm{FEV}_{1}$} \\
\hline Diabetes-Yes & $3.0 \pm 0.8$ & $0.38(0.28,0.52)$ & & & \\
\hline Diabetes-No & $3.8 \pm 0.9$ & 1.00 (ref) & & & \\
\hline \multicolumn{6}{|l|}{$\mathrm{FEV}_{1} / \mathrm{FVC}$ ratio $\%$} \\
\hline Diabetes-Yes & $80.5 \pm 7.4$ & $0.97(0.94,0.99)$ & & & \\
\hline Diabetes-No & $82.7 \pm 7.2$ & 1.00 (ref) & & & \\
\hline
\end{tabular}

${ }^{\mathrm{a}}$ This included heart disease, stroke and leg ulcers or amputations

${ }^{\mathrm{b}}$ This included eye sight problems and kidney problems

'This included chronic bronchitis, emphysema and chronic obstructive pulmonary disease (COPD) 
Table $\mathbf{5}$ ORs of diabetes risk based on multivariate logistic regression analysis

\begin{tabular}{|c|c|c|c|c|c|}
\hline \multicolumn{3}{|l|}{ Model with BMI } & \multicolumn{3}{|l|}{ Model without BMI } \\
\hline Parameter & Odds Ratio (95 \% Cl) & $P$ value & Parameter & Odds Ratio $(95 \% \mathrm{Cl})$ & $P$ value \\
\hline Sex & & & Sex & & \\
\hline Female & $1.21(0.78,1.88)$ & 0.397 & Female & $1.54(1.00,2.38)$ & 0.050 \\
\hline Male & 1.00 (ref) & & Male & 1.00 (ref) & \\
\hline Age & & & Age & & \\
\hline $50+$ years & $8.72(4.62,16.46)$ & $<0.0001$ & $50+$ years & $12.82(6.94,23.67)$ & $<0.0001$ \\
\hline 40-49 years & $3.62(1.78,7.35)$ & $<0.0001$ & 40-49 years & $5.15(2.58,10.27)$ & $<0.0001$ \\
\hline 30-39 years & $2.44(1.19,4.99)$ & 0.015 & 30-39 years & $3.28(1.63,6.58)$ & 0.001 \\
\hline 17-29 years & 1.00 (ref) & & $17-29$ years & 1.00 (ref) & \\
\hline \multicolumn{6}{|l|}{ Body Mass Index } \\
\hline Obese & $8.97(3.58,22.52)$ & $<0.0001$ & & & \\
\hline Overweight & $3.86(1.51,9.87)$ & 0.005 & & & \\
\hline Normal & 1.00 (ref) & & & & \\
\hline Past year duration in reserve & & & Past year duration in reserve & & \\
\hline All 12 months & $1.69(0.96,2.97)$ & 0.068 & All 12 months & $1.83(1.05,3.18)$ & 0.032 \\
\hline Less than 12 months & 1.00 (ref) & & Less than 12 months & 1.00 (ref) & \\
\hline Ever experienced discrimination & & & Ever experienced discrimination & & \\
\hline High (8-11) & $0.11(0.02,0.54)$ & 0.007 & High (8-11) & $0.12(0.03,0.54)$ & 0.006 \\
\hline Moderate (4-7) & $1.23(0.70,2.16)$ & 0.464 & Moderate (4-7) & $1.24(0.73,2.13)$ & 0.428 \\
\hline Little (0-3) & 1.00 (ref) & & Little (0-3) & 1.00 (ref) & \\
\hline Model with Abdominal Girth & & & Model without Abdominal Girth & & \\
\hline Parameter & Odds Ratio (95 \% Cl) & $P$ value & Parameter & Odds Ratio (95\% Cl) & $P$ value \\
\hline Sex & & & Sex & & \\
\hline Female & $1.01(0.64,1.58)$ & 0.978 & Female & $1.54(1.00,2.38)$ & 0.050 \\
\hline Male & 1.00 (ref) & & Male & 1.00 (ref) & \\
\hline Age & & & Age & & \\
\hline $50+$ years & $9.87(5.29,18.41)$ & $<0.0001$ & $50+$ years & $12.82(6.94,23.67)$ & $<0.0001$ \\
\hline 40-49 years & $4.12(2.01,8.46)$ & $<0.0001$ & 40-49 years & $5.15(2.58,10.27)$ & $<0.0001$ \\
\hline 30-39 years & $2.64(1.28,5.46)$ & 0.009 & 30-39 years & $3.28(1.63,6.58)$ & 0.001 \\
\hline $17-29$ years & 1.00 (ref) & & $17-29$ years & 1.00 (ref) & \\
\hline \multicolumn{6}{|l|}{ Abdominal Girth } \\
\hline High risk & $5.87(2.67,12.92)$ & $<0.0001$ & & & \\
\hline Not available & $4.40(1.75,11.07)$ & 0.002 & & & \\
\hline Low risk & 1.00 (ref) & & & & \\
\hline Past year duration in reserve & & & Past year duration in reserve & & \\
\hline All 12 months & $1.84(1.05,3.20)$ & 0.032 & All 12 months & $1.83(1.05,3.18)$ & 0.032 \\
\hline Less than 12 months & 1.00 (ref) & & Less than 12 months & 1.00 (ref) & \\
\hline Ever experienced discrimination & & & Ever experienced discrimination & & \\
\hline High (8-11) & $0.11(0.02,0.49)$ & 0.004 & High (8-11) & $0.12(0.03,0.54)$ & 0.006 \\
\hline Moderate (4-7) & $1.11(0.63,1.93)$ & 0.717 & Moderate (4-7) & $1.24(0.73,2.13)$ & 0.428 \\
\hline Little (0-3) & 1.00 (ref) & & Little (0-3) & 1.00 (ref) & \\
\hline
\end{tabular}

of discrimination experienced a significantly lower risk of diabetes (OR 0.11; $95 \%$ CI 0.02, 0.54) after adjusting for other key variables such as sex, BMI (or waist circumference) and age group. Removal of adiposity measures from the analysis did not affect this finding. However, we additionally looked for possible trends in 
other variables that might explain the inverse relationship between diabetes and perceptions of discrimination (data not shown). Compared to those with the lowest levels of perceived discrimination, a larger proportion of people with the highest levels of perceived discrimination were employed or students (41\% versus $34.1 \%$; $p=$ 0.791 ), married or common-law (53\% versus $37.3 \%$; $p=$ 0.007 ), had household incomes $>\$ 20,000$ (38.6\% versus $23.1 \% ; p=0.022)$, and had attended residential schools $(68.7 \%$ versus $35.7 \% ; p<0.0001)$.

\section{Discussion}

The prevalence of diabetes among adults in two Saskatchewan First Nations communities was higher than that reported for the province's non-First Nations population [2], as well as for a predominantly Caucasian subgroup living in rural Saskatchewan during a similar time period [30]. As expected, we found that increasing age and body weight were strong predictors of diabetes in these First Nations communities. We again confirmed the marked predilection of diabetes for First Nations females [2] and have now shown that this is at least partly explained by increased adiposity. With respect to the primary objective of the study, we were unable to show at the individual level that residential school attendance by either study participants or their parents/grandparents significantly increased diabetes risk. The same was true for inability to speak Cree and/or low participation in cultural events. The most unique finding of this study was that those experiencing the highest levels of discrimination at an individual level had the lowest prevalence of diabetes.

As far as we are aware, this is the first study that has examined the relationship between individual-level diabetes risk and factors related to colonization within First Nations communities, while adjusting for a comprehensive set of other important and recognized predictors of diabetes. In addition to female gender, increasing age, higher BMI and elevated waist circumference, known diabetes risk factors include social determinants of health $[17,18]$ such as poorer income, employment status, education and quality of housing. In our study, better housing and being employed or engaged as a student were associated with lower diabetes prevalence on univariate analysis, but did not remain significant in the final model. Higher household income adequacy and education were not protective for diabetes before or after adjusting for other variables. However, it is possible that we did not sufficiently discriminate between the highest and lowest levels of education and income, and/ or that participant numbers in the study did not afford us the statistical power to show a difference. With respect to life style, univariate analysis showed a trend towards lower diabetes prevalence among those who engaged in physical activity, and there were lower rates of diabetes among those who currently smoked or consumed alcohol on a regular basis. In other studies, alcohol intake has been shown to protect against diabetes [31] while smoking is an important diabetes risk factor [32]. Since neither activity remained in the final model in this study, it is possible that our univariate findings were related to higher alcohol intake and smoking rates among younger people who have a much lower prevalence of diabetes.

Results of qualitative research have consistently identified various dimensions of cultural continuity/disruption as important to the health of Indigenous Canadians [33, 34]. However, the results of quantitative research on this topic are mixed. On the one hand, several quantitative studies have demonstrated associations between greater cultural continuity and better mental health outcomes $[35,36]$. For example, Chandler and Lalonde have shown that cultural continuity in First Nations communities is protective against youth suicide [37, 38]. In addition, preliminary research conducted as part of the larger study from which our data is drawn, also shows a significant relationship between discriminatory experiences and compromised mental health among First Nations adults [39]. However, a number of other quantitative studies in Canada have reported either no or mixed/contradictory associations between indicators of cultural continuity and health, depending upon the health outcome and particular aspect of culture under study [40-42]. With respect to metabolic disorders, there is only one study that suggests that cultural continuity may be protective against diabetes for First Nations people [43]. After adjusting for differences in income, employment rates and education in 31 First Nations communities in Alberta, Oster and colleagues recently reported on an ecologic study that showed lower diabetes prevalence in those communities that had a greater retention of Indigenous language.

In this study, lack of participation in cultural events and low scoring of community strengths was neither predictive nor protective for diabetes on an individual level. People who spoke Cree were over 3 times more likely to have diabetes than others on univariate analysis but this did not remain significant in the final model. It is likely that the univariate finding was due to a larger proportion of older people with a knowledge of Cree, since increasing age is also a strong predictor of diabetes. It should be emphasized that an important difference between this study and that of Oster et al. mentioned above [43], is that we examined the individual-level risk of diabetes by his/her knowledge of Cree. In contrast, Oster et al. examined the relationship between diabetes prevalence and Indigenous language knowledge at the community level. At face value, these findings may appear contradictory. However, had they 
looked at within-community data (and regardless of a particular community's prevalence of diabetes), it is quite possible that Oster et al. would have also found a higher proportion of older individuals with diabetes, and it is also quite possible that older diabetic individuals were more likely to speak an Indigenous language than their younger counterparts.

Although study participants who had attended residential school had a slightly higher prevalence of diabetes than those who had not, the finding was not statistically significant. Furthermore, while those whose parents or grandparents had attended residential school trended towards a lower prevalence of diabetes than participants without that family history, the difference was also not significant. Because of the stressful and often traumatic experiences that many residential school survivors report [24], as well as the intra- and inter-generational impact of those experiences [44], we had anticipated that residential school attendees and their offspring might have a higher risk for diabetes. It is known that stress can be diabetogenic through many pathways [22] including endocrine mechanisms [45] and by causing chromosomal damage [46]. However, it is possible that the detrimental impact of residential schools on the health of individuals within First Nations communities is so pervasive that it is difficult to find a truly unexposed comparison group. Furthermore, there are many additional causes of stress within First Nations communities and it may not be possible to readily differentiate between the relative importance of residential school exposure and other stress-producing experiences. Larger study populations and/or comparison with nonFirst Nations groups truly unexposed to the impact of residential schools should be considered in future research examining this question.

We had also speculated that those experiencing the most discrimination would have a higher prevalence of diabetes, once again because of increased stress. Instead, we found the opposite. The extent to which people perceived discrimination during day-to day experiences was inversely related to diabetes prevalence, and this highly significant finding was present even after adjusting for other important independent variables. What could explain this relationship? It was not because of characteristics most clearly associated with lower diabetes prevalence such as younger age, being male and having normal adiposity. It also seems unlikely that avoidance of the health care system and delay of diabetes diagnosis could explain such a significant finding. Rather, the main thread that appears to connect those within this group is that they were experiencing negative interactions in what were predominantly non-Aboriginal settings. Indeed, our multivariate model results showed that spending time away from the reserve was protective for diabetes. Finally, and consistent with recent research involving
Indigenous Australian adults $[47,48]$, we also observed that people experiencing the most discrimination (and lowest diabetes prevalence) tended to be employed or students, and were significantly more likely to be married and to have higher household incomes. It is therefore possible that experiencing discrimination is a proxy measure for being engaged in and with society offreserve. That engagement may lead to improvements in the social determinants of health - including higher socio-economic status - and engender physical health benefits, at least with respect to diabetes risk. As discussed above, however, this comes at a high emotional price via increased experiences of interpersonal discrimination [39]. This is perhaps the most important finding of our study and requires further reflection as well as corroboration to fully understand its implications. As with the impact of residential schools, comparison of First Nations with non-First Nations populations, while adjusting for social determinants of health, could clarify the links between discrimination and physical health outcomes like diabetes.

First Nations people with diabetes were more likely than others to experience a number of co-morbidities and to evaluate their general health as fair or poor. Because the primary focus of the main study was on lung health [23], much of the questionnaire pertained to pulmonary conditions, and many significant associations of these with diabetes were present on univariate analysis. While none were significant in initial multivariate models that included co-morbidities (perhaps largely due to age adjustment), it is likely that obstructive sleep apnea is common among First Nations people with diabetes because obesity is an important risk factor for both [49]. Not surprisingly, both macro and micro vascular complications were very common among those with diabetes and these relationships remained highly significant in initial multivariate models that included comorbidities. Interestingly, women with diabetes were less likely than men to have micro-vascular complications like kidney and eye disease. This is consistent with other research that shows a higher cumulative incidence of end stage renal disease among First Nations men [4].

Strengths of this study included the community-based participatory research model used, the large proportion of eligible adults and households that participated, and our ability to investigate the potential role of both known and unrecognized variables in understanding the disproportionate risk for diabetes experienced by First Nations communities. Accordingly, this is the first study that we are aware of to examine the potential role of factors related to colonization in diabetes risk while at the same time adjusting for a comprehensive set of other known diabetes predictors. Limitations include the cross sectional nature of the study and the associated inability 
to be confident about cause-effect relationships. To limit the length of the questionnaire, we omitted questions on important predictors of diabetes such as family history. Although the sample size was sufficient for the main study, the relatively small number of people with diabetes in this sub-study may have prevented us from demonstrating some relationships that truly exist, most importantly the impact of residential school attendance on diabetes risk. The discrimination scale that was used reflects racism on a personal level and we were not able to study the impact of other forms of racism [50] on diabetes risk. For example, it is quite possible that some elements of systemic racism experienced by First Nations people might be more clearly associated with diabetes risk. This reinforces the need for more research focusing on quantitative measurements of cultural disruption, residential school impact and racism among Indigenous peoples. Finally, while our sample had an equal gender distribution, it contained a relatively larger proportion of older people compared to the overall Saskatchewan First Nations population [51]. Therefore, we may not be able to generalize our findings to other First Nations communities.

\section{Conclusions}

Increased diabetes prevalence in two Saskatchewan First Nations communities was significantly related to known diabetes risk factors including female sex, increasing age and BMI but we were unable to demonstrate in this study that diabetes was associated with residential school exposure, perceptions of community strength or inability to speak Cree. An unanticipated finding was that those experiencing the most discrimination had the lowest prevalence of diabetes. While the reasons underlying this latter finding remain unclear, engagement with society off-reserve may have certain physical health benefits for First Nations people that relate to improved socioeconomic status and other undefined influences. However, our findings suggest that better physical health comes at a high emotional price via increased experiences of interpersonal discrimination [39]. While this important observation may apply to other Indigenous peoples in Canada and elsewhere, it requires corroboration in diverse jurisdictions. Additional dialogue with the communities as well as further reflection and indepth analysis will help to fully understand its implications.

\section{Availability of data and materials}

The data used in the analysis is kept in a secure location within the Canadian Center for Health and Safety in Agriculture, University of Saskatchewan. As part of the contractual agreement with the two First Nations communities involved in the study, the data is not publicly available.

\section{Competing interests}

The authors declare that they have no competing interests.

\section{Authors' contributions}

RD conceived of the study, participated in its design and analysis, and drafted the manuscript. CK participated in the design of the study and carried out the statistical analysis. BJ, JL, VRR, DR, and SA are co-investigators on the research grant, helped design the study, and reviewed and revised the manuscript for important intellectual content. JPG and LM provided community input into the design of the study, helped with acquisition of data, and reviewed and revised the manuscript from a community perspective. JD, JAE and PP are co-principal investigators on the research grant, helped design the study, and reviewed and revised the manuscript for important intellectual content. All authors read and approved the final manuscript.

\section{Acknowledgements}

This study was funded by a grant from the Canadian Institutes of Health Research "Assess, Redress, Re-assess: Addressing Disparities in Respiratory Health among First Nations People", CIHR MOP-246983-ABH-CCAA-11829. The First Nations Lung Health Project Team consists of: James Dosman, MD (Designated Principal Investigator, University of Saskatchewan, Saskatoon, SK Canada); Dr. Punam Pahwa, PhD (Co-principal Investigator, University of Saskatchewan, Saskatoon SK Canada); Jo-Ann Episkenew, PhD (Co-principal Investigator, Indigenous People's Health Research Centre, University of Regina, SK Canada), Sylvia Abonyi, PhD (Co-principal Investigator, University of Saskatchewan, Saskatoon, SK Canada); Co-Investigators: Mark Fenton, John Gordon, Bonnie Janzen, Chandima Karunanayake, Malcolm King, Shelly Kirychuk, Niels Koehncke, Joshua Lawson, Greg Marchildon, Lesley McBain, Donna Rennie, Vivian R Ramsden, Ambikaipakan Senthilselvan; Collaborators: Amy Zarzeczny, Louise Hagel, Breann Davis, John Dosman, Roland Dyck, Thomas Smith-Windsor, William Albritton; External Advisor: Janet Smylie; Project Manager: Kathleen McMullin; Community Partners: Jeremy Seeseequasis, P. Jenny Gardipy, Candace Scott, Laurie Jimmy, Laura McCallum, Jarret Nelson. We are grateful for the contributions of community leaders included in the study that facilitated the engagement necessary for the study and to all of the participants who donated their time and expertise to participate in the survey.

\section{Author details}

${ }^{1}$ Canadian Centre for Health and Safety in Agriculture, College of Medicine, University of Saskatchewan, 104 Clinic Place, Saskatoon, Saskatchewan S7N 2Z4, Canada. ${ }^{2}$ Department of Medicine, University of Saskatchewan, Saskatoon, Saskatchewan, Canada. ${ }^{3}$ Department of Community Health and Epidemiology, University of Saskatchewan, Saskatoon, Saskatchewan, Canada. ${ }^{4}$ Department of Academic Family Medicine, University of Saskatchewan, Saskatoon, Saskatchewan, Canada. ${ }^{5}$ Willow Cree Health Centre, Duck Lake, Saskatchewan, Canada. ${ }^{6}$ William Charles Health Centre, Montreal Lake Cree Nation, Saskatchewan, Canada. 'Indigenous Peoples' Health Research Centre, University of Regina, Regina, Canada.

Received: 11 September 2015 Accepted: 30 November 2015 Published online: 09 December 2015

\section{References}

1. Young TK, Reading J, Elias B, O'Neil J. Type 2 diabetes mellitus in Canada's First Nations: status of an epidemic in progress. Can Med Assoc J. 2000;163(5):561-6.

2. Dyck RF, Osgood N, Lin TH, Gao A, Stang MR. Epidemiology of diabetes mellitus among First Nations and non-First Nations adults. Can Med Assoc J. 2010;182(3):249-56.

3. Dyck RF, Osgood ND, Gao A, Stang MR. The epidemiology of diabetes mellitus among First Nations and non-First Nations children in Saskatchewan. Can J Diabetes. 2012;36:19-24.

4. Jiang Y, Osgood N, Lim HJ, Dyck RF. Differential Mortality and the excess burden of end-stage renal disease among First Nations people with diabetes; a competing risks analysis. Can Med Assoc J. 2014;186(2):103-9. 
5. Dyck RF, Osgood ND, Lin TH, Gao A, Stang MR. End-stage renal disease in people with diabetes: a comparison of First Nations people and other Saskatchewan residents from 1981-2005. Can J Diab. 2010;34(4):324-33.

6. Dyck RF, Naqshbandi Hayward M and Harris SB. Prevalence, Predictors and Co-Morbidities of Chronic Kidney Disease among First Nations Adults with Diabetes: Results from the CIRCLE study. BMC Neph. July 2012.13.57; doi:10.1186/1471-2369-13-57

7. Deved V, Jette N, Quan H, Tonelli M, Manns B, Soo A, Barnabe C, Hemmelgarn BR for the Alberta Kidney Disease Network. Quality of care for First Nations and non-First Nations people with diabetes. CJASN. February 2013; doi:10.2215/CJN.10461012

8. Harris SB, Naqshbandi M, Bhattacharyya O, Hanley AJG, Esler JG, Zinman B, et al. Major Gaps in Diabetes Clinical Care among Canada's First Nations: Results of the CIRCLE Study. Diabetes Res Clin Pract. 2011;92:272-9.

9. Barroso I. Genetics of type 2 diabetes. Diab Med. 2005;22:517-35.

10. Herman $W H$, Zimmet $P$. Type 2 diabetes: an epidemic requiring global attention and urgent action. Diab Care. 2012;35:943-4.

11. Drong AW, Lindgren CM, McCarthy MI. The genetic and epigenetic basis for type 2 diabetes. Clin Pharm Ther. 2012;92(6):707-15.

12. Zimmet PZ, Magliano DJ, Herman WH, Shaw JE. Diabetes: a 21st century challenge. Lancet Diabetes Endocrinol. 2013;2:56-64.

13. Naser KA, Gruber A, Thomson GA. The emerging disaster of obesity and diabetes: are we doing enough to prevent a disaster? Int J Clin Pract. 2006;60(9):1093-7.

14. Katzmarzyk PT, Malina RM. Obesity and relative subcutaneous fat distribution among Canadians of First Nation and European ancestry. Int J Obesity. 1998;22:1127-31.

15. Feig DS, Zinman B, Wang X, Hux JE. Risk of development of diabetes mellitus after diagnosis of gestational diabetes. Can Med Assoc J. 2008;179(3):229-34.

16. Dabalea D, Pettitt DJ. Intrauterine diabetic environment confers risk for type 2 diabetes mellitus and obesity in the offspring, in addition to genetic susceptibility. J Pediatr Endocrinol Metab. 2001;14:1085-91.

17. Mikkonen J, Raphael D. Social determinants of health: the Canadian facts. Toronto: York University of Health Policy and Management; 2010.

18. Agardh E, Allebeck P, Hallqvist J, Moradi T, Sidorchuk A. Type 2 diabetes incidence and socio-economic position: a systematic review and metaanalysis. Int J Epidemiol. 2011;40(3):804-18. doi:10.1093/ije/dyr029

19. Reading $\mathrm{CL}$, Wien $\mathrm{E}$. Health Inequalities and Social Determinants of Aboriginal Peoples' Health. National Collaborating Centre for Aboriginal Health. 2009

20. King M, Smith A, Gracey M. Indigenous health part 2: the underlying causes of the health gap. Lancet. 2009;374:76-85.

21. Barsh R. Canada's Aboriginal People: Social integration or disintegration? Can J Native Studies. 1994;14(1):1-46.

22. Pascoe EA, Smart-Richman L. Perceived discrimination and health: a metaanalytic review. Psychological Bulletin. 2009;135(4):531-54

23. Pahwa P, Abonyi S, Karunanayake C, Rennie D, Janzen B, Kirychuk S, et al. A community-based participatory research methodology to address, redress and reassess disparities in respiratory health among First Nations. BMC Res Notes. 2015:8:199. doi:10.1186/s13104-015-1137-5.

24. Milloy J. A National Crime: The Canadian Government and the Residential School System, 1879 to 1986. Winnipeg: University of Manitoba Press; 1999.

25. Krieger N. Racial and gender discrimination: risk factors for high blood pressure? Soc Sci Med. 1990;30:1273-81.

26. Krieger N, Sidney S. Racial discrimination and blood pressure: the CARDIA study of young black and white adults. AJPH. 1996:86:1370-8

27. Krieger N, Smith K, Naishadham D, Hartman C, Barbeau EM. Experiences of discrimination: validity and reliability of a self-report measure for population health research on racism and health. Soc Sci Med. 2005;61:1576-96.

28. Pan $\mathbf{W}$. Akaike's information criterion in generalized estimating equations. Biometrics. 2001;57(1):120-5.

29. Hardin JW, Hilbe JM. Generalized Estimating Equations. New York, NY: Chapman \& Hall/CRC; 2003.

30. Dyck RF, Karunanayake CP, Pahwa P, Hagel L, Lawson JA, Dosman JA, et al. Prevalence, risk factors and co-morbidities of diabetes among adults in rural Saskatchewan: the influence of farm residence and agriculture-related exposures. BMC Public Health. 2013;13:7. doi: 10.1186/1471-2458-13-7.

31. Koppes LLJ, Dekker JM, Hendriks HFJ, Bouter LM, Heine RJ. Moderate alcohol consumption lowers the risk of type 2 diabetes: A meta-analysis of prospective observational studies. Diabetes Care. 2005;28(3):719-25.
32. Smoking and diabetes, Willi C, Bodenmann P, Ghali WA, Faris PD, Cornuz J. Active smoking and the risk of type 2 diabetes: a systematic review and meta-analysis. JAMA. 2007;298(22):2654-64.

33. Richmond CA, Ross NA. The determinants of First Nation and Inuit health: a critical population health approach. Health Place. 2009;15(2):403-11.

34. Brown HJ, McPherson G, Peterson R, Newman V, Cranmer B. Our land, our language: connecting dispossession and health equity in an indigenous context. CJNR. 2012;44(2):44-63.

35. Hallett D, Chandler MJ, Lalonde C. Aboriginal language knowledge and youth suicide'. Cogn Dev. 2007;22(3):392-99.

36. Currie CL, Wild TC, Schopflocher DP, Laing L, Veugelers P. Illicit and prescription drug problems among urban Aboriginal adults in Canada: The role of traditional culture in protection and resilience. Soc Sci Med. 2013;88:1-9.

37. Chandler MJ, Lalonde C. Cultural continuity as a hedge against suicide in Canada's first Nations. Transcult Psychiatry. 1998;35(2):191-219.

38. Chandler MJ, Lalonde C. Cultural continuity as a protective factor against suicide in First Nations youth - A special issue on Aboriginal youth: Hope or heartbreak, Aboriginal youth and Canada's future. Horizons. 2003;10(1):68-72.

39. Janzen B, Episkenew JA, Abonyi S, Karunanayake C, Seeseequasis J, Naytowhow A, Dosman JA, Pahwa P and the First Nations Lung Health Team. Self-reported discrimination and mental health among First Nations people in Canada. SHARP Conference, Saskatoon Canada, October 2014. (Abstract)

40. Bombay A, Matheson K, Anisman H. Decomposing identity: differential relationships between several aspects of ethnic identity and the negative effects of perceived discrimination among First Nations adults in Canada. Cultur Divers Ethnic Minor Psychol. 2010;16(4):507.

41. Wilson K, Rosenberg MW. Exploring the determinants of health for First Nations peoples in Canada: can existing frameworks accommodate traditional activities? Soc Sci Med. 2002;55(11):2017-31.

42. Wilson K, Cardwell N. Urban Aboriginal health: Examining inequalities between Aboriginal and non-Aboriginal populations in Canada. The Canadian Geographer/Le Géographe Canadien. 2012;56(1):98-116.

43. Oster RT, Grier A, Lightening R, Mayan MJ, Toth EL. Cultural continuity, traditional Indigenous language, and diabetes in Alberta First Nations: a mixed methods study. Int J Equity Health. 2014;13(92):1-11.

44. Smith D, Varcoe C, Edwards N. Turning around the intergenerational impact of residential schools on Aboriginal people: implications for health policy and practices. CJNR. 2005;7(4):38-60.

45. Pervanidou P, Chrousos GP. Metabolic consequences of stress during childhood and adolescence. Metabol. 2012;61:611-9.

46. Shalev I, Moffitt TE, Sugden K, Williams B, Houts RM, Danese A, et al. Exposure to violence during childhood is associated with telomere erosion from 5 to 10 years of age: a longitudinal study. Mol Psychiatry. 2013;18(5):576-81. doi:10.1038/mp.2012.32.

47. Paradies YC, Cunningham J. Experiences of racism among urban Indigenous Australians: Findings from the DRUID study. Ethnic and Racial Studies. 2009;32(3):548-73.

48. Cunningham J, Paradies YC. Patterns and correlates of self-reported racia discrimination among Australian Aboriginal and Torres Strait Islander adults, 2008-09: analysis of national survey data. Int J Equity Health. 2013;12:47.

49. Tasali E, Mokhlesi B, Van Cauter E. Obstructive sleep apnea and type 2 diabetes: interacting epidemics. Chest. 2008;133(2):496-506.

50. Allan B, Smylie J. First Peoples, second class treatment: The role of racism in the health and well-being of Indigenous peoples in Canada. Toronto ON: the Wellesley Institute; 2015.

51. Statistics Canada. Saskatchewan Aboriginal Peoples, 2006. Census of Canada: Statistics Canada; 2008. 